\title{
Interferometric Investigations of Eclipsing Binaries as a Key to an Improved Distance Scale
}

\author{
K. Shabun ${ }^{1}$, A. Richichi ${ }^{1}$, U. Munari ${ }^{2}$, A. Siviero ${ }^{2}$, and B. Pacsysnki ${ }^{3}$ \\ ${ }^{1}$ ESO, European Southern Observatory \\ email: kshabun@eso.org \\ ${ }^{2}$ INAF, Osservatorio Astronomico di Padova \\ ${ }^{3}$ Princeton University
}

\begin{abstract}
Binary and multiple systems constitute one of the main tools for obtaining fundamental stellar parameters, such as masses, radii, effective temperatures and distances. One especially fortunate, and at the same time rare, occurrence is that of double-lined eclipsing binaries with well-detached components. In this special case, it is possible to obtain a full solution of all orbital and stellar parameters, with the exception of the effective temperature of one star, which is normally estimated from spectral type or derived from atmospheric analysis of the spectrum. Long-baseline interferometry at facilities such as the ESO VLTI is beginning to have the capability to measure directly the angular separation and the angular diameter of some selected eclipsing binary systems, and we have proposed such observations with the AMBER instrument. In particular, we aim at deriving directly the effective temperature of at least one of the components in the proposed system, thereby avoiding any assumptions in the global solution through the Wilson-Devinney method. We will also obtain an independent check of the results of this latter method for the distance to the system. This represents the first step towards a global calibration of eclipsing binaries as distance indicators. Our results will also contribute to the effective temperature scale for hot stars. The extension of this approach to a wider sample of eclipsing binaries could provide an independent method to assess the distance to the LMC. The observations will extend accurate empirical calibration to spectral type O9 - B0.
\end{abstract}

Keywords. Eclipsing binaries, interferometry, distance determination

\section{Introduction}

A significant step forward in this area could be made if one could measure directly, in addition to the available light curves and radial velocity, also the angular semi-major axis and the angular diameters of the two stars $\left(a, \theta_{1}\right.$, and $\left.\theta_{2}\right)$ of an eclipsing binary $(\mathrm{EB})$ system. In this case, one could have an independent and precise determination of the distance (from the combination of $a$ with the radial velocity data) to be compared with the WD solution. At the same time, the combination of $\theta_{1}$ and $\theta_{2}$ with the bolometric fluxes (from photometry out of the eclipses) would yield a direct estimate of the effective temperature, thus removing the only undetermined parameter in the WD solution. Because separations are usually equal to a few stellar diameters, and the distances to the objects are large, it is difficult to measure the angular separations and diameters directly and this has never been accomplished until now. We propose, for the first time, to use interferometry in order to obtain a full solution of some eclipsing binary systems. The angular semi-major axis and the angular diameters $\left(a, \theta_{1}\right.$, and $\left.\theta_{2}\right)$ will be measured with AMBER at the VLTI using baselines of $<100 \mathrm{~m}$ in the near-infrared, provided they are close to the milliarcsecond (mas) scale: favorable cases would be represented by EBs with 


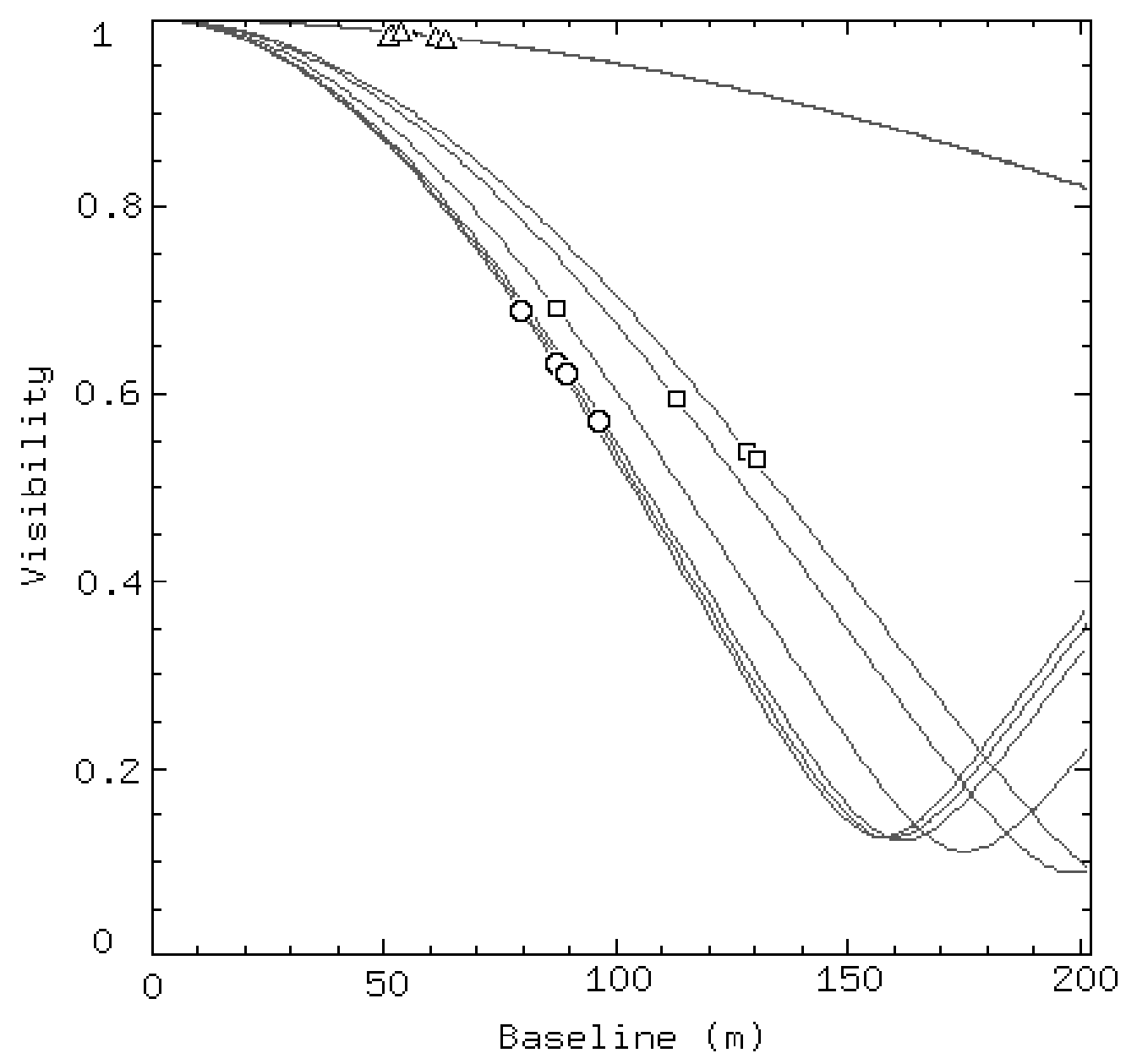

Figure 1. Simulations of the visibilities of $\delta$ Ori, to be observed with AMBER UT1-UT3-UT4, using a two circular disk model. Each dot shape corresponds to a different baseline, and four triplets (one per observation) are present. We will also obtain one closure phase per observation.

$a$ in the range $1-5$ mas, and $\theta_{1}, \theta_{2} 0.4-1.2$ mas. Time has been granted to observe $\delta$ Ori, $a=1.4, \theta_{1}=0.98, \theta_{2}=0.55$ (distance $280 \mathrm{pc}$ ), during ESO Period 78 (starting in October 2006).

\section{Method}

We stress that this observational breakthrough is made possible for the first time by the combination of the long VLTI baselines with the accuracy, sensitivity and wavelength range of the UT+AMBER. Full solutions (masses, radii, effective temperatures, distances) will be initially obtained for the proposed objects. One of the possible applications of the completed calibration is the precise determination of the distance to the Large Magellanic Cloud. Eclipsing binaries have been used in this sense already, however the results have been based on model assumptions and subject to uncertainties in reddening. Our system is considerably closer and the observations will be made in the near-IR, hence extinction will be only a minor problem for the interferometric measurements and 
we will derive model-independent effective temperatures. If our initial observations are successful, we plan to expand them to a wider sample of binaries with diverse spectral types. This task will be facilitated by the availability of the $J$-band with AMBER, currently undergoing commissioning, and the possible opening of AMBER operation on AT baselines which are longer than the maximum UT combination. This combination will provide a further factor of two improvement in angular resolution. We thus aim at an extended calibration that could be used to solve the uncertainty in the distance scale to the LMC. We propose to use AMBER, currently offered on UTs and $H-K$ range only, in the combination that provides the highest angular resolution, i.e., triplets which include the UT1-UT4 baseline and the low-resolution LR-HK mode. This mode provides dispersed visibilities over the $1.5-2.4 \mu \mathrm{m}$ range: the shorter wavelengths will provide the best angular resolution for the individual diameters, but the whole range can be used for the angular separation and also to provide a solid estimate of the brightness ratio as a function of the wavelength. This mode also provides the best SNR: although the candidate EB system is sufficiently bright and we will be able to provide comparably bright calibrators, a good SNR will be essential to ensure sufficient quality of the results.

Each observation will provide 3 visibilities and one closure phase for the system. We will thus be able to determine the separation and the orientation of the system. We propose to do this at the time of maximum separation. In order to improve the precision of the observed quantities, the observation will be repeated four times (i.e., two times at opposite phases), thus providing a reduction of about a factor of two on the final errors with respect to a single measurement. At the end of the four separate observations, we will have 12 visibilities and 4 closure phases. The quantities to be fitted are the (fixed) angular diameters, the (fixed) brightness ratio, and four pairs of angular separation and position angle values. Thus, the system can be solved. Also note that we already have good estimates for each of these parameters, and that the period and photometric phases are very well known. We have already performed simulations of the problem using realistic values, and are confident to be able to retrieve the correct solutions. The results of these simulations are shown in Figure 1 above.

\section{Conclusion}

We have investigated various lists of EB systems to identify a candidate suitable in terms of near-IR brightness, angular separation, angular diameters, and period. Additionally, we have put an emphasis on systems relatively close to the celestial equator in order to take advantage of the possibility offered by the Asiago observational facilities for obtaining new and accurate photometric and radial velocity curves able to provide uncertainties $1 \%$ on masses, radii and semi-major axes for the selected systems. Support high quality radial velocity and photometric light-curve for the proposed targets are currently being collected at Asiago Observatory.

\section{References}

Paczynski, B. 1997, Space Tel. Sc. Inst. p. 273

Munari, U., Siviero, A. et al. 2004, A\&SA 418, L31

Siviero A., Munari U. et al. 2004, A\&\&A 417, 1083

Richichi, A. et al. 2005, A\&SA 431, 773

Richichi, A., Percheron, I. 2005, A\&A 434, 1201 